\title{
Barriers to Implementing a Pharmacist-Run Immunization Service, as Perceived by community Pharmacists in a Middle Eastern Country: A cross sectional web-based survey
}

Dalal Youssef ( $\nabla$ dalalyoussef.esu@gmail.com )

Ministry of Public Health

Linda Abou Abbas

Ministry of Public Health

Hamad Hassan

Ministry of Public Health

\section{Research Article}

Keywords: Barriers, implementation, Pharmacist-Run, Immunization, Middle Eastern Country

Posted Date: September 1st, 2021

DOI: https://doi.org/10.21203/rs.3.rs-861898/v1

License: (c) (i) This work is licensed under a Creative Commons Attribution 4.0 International License.

Read Full License 


\section{Abstract}

Background: Several barriers to instigating a pharmacist-run immunization program existed. This survey aims to identify the barriers perceived by Lebanese community pharmacists (CPs) to implementing such a program and the association of these barriers with their willingness to provide immunization services.

\section{Methods:}

A cross-sectional study using an online survey was conducted over the period of time extending between the $1^{\text {st }}$ of November and the end of December 2020 among Lebanese community pharmacists. The questionnaire included information on their socio-demographic characteristics, willingness to vaccinate and the perceived barriers to the run of immunization service. Statistical analyses were performed using SPSS version 24.0. Two logistic regression were conducted, one to identify factors associated with pharmacists high perception of barriers and another one to determine the predictors of the pharmacist's willingness to vaccinate.

Results:

Out of the 412 surveyed community pharmacists, $67.48 \%$ of them were willing to expand their practice scope to include immunization. The main perceived barriers were the conflict with other professionals, liability, and reimbursements concerns, time for professional development and lack of knowledge of adverse events after immunization. Our results showed that females were more likely to perceive barriers than males $(\mathrm{aOR}=2.460,95 \% \mathrm{Cl}(1.876: 6.909))$. However, pharmacists reporting previous experience in immunization (aOR=0.250,95\% Cl (0.170:0.586)), working in urban area $(\mathrm{aOR}=0.451,95 \% \mathrm{Cl}$ (0.199:0.926) ) and having extensive working hours (aOR=0.244, $95 \% \mathrm{Cl}(0.05: 1.2))$ were less likely to perceive barriers. Female pharmacists were less willing to vaccinate $(\mathrm{aOR}=0.211,95 \% \mathrm{Cl}(0.116: 0.383)$ ). However, having less than 40 years old $(\mathrm{aOR}=2.518,95 \% \mathrm{Cl}(1.236: 5.130))$, having large experience ((aOR=1.78, $95 \% \mathrm{Cl}(1.087$ to 2.993$)$ ) or previous experience in immunization (aOR=1.702, 95\% $\mathrm{Cl}(1.092$ to 3.141)), being pharmacy owner ( $\mathrm{aOR}=1.504,95 \% \mathrm{Cl}(1.098: 2.103))$ and working more than 24 hours per week (aOR=2.622, 95\% Cl (1.447:4.752) were positively associated to the willingness to vaccinate. Pharmacists who perceived reimbursement (aOR=0.449 with $95 \% \mathrm{Cl}(0.264-0.765))$, lack of pharmacy space (aOR $=0.410$ with $95 \% \mathrm{Cl}(0.158-1.060))$, sharp safety (aOR=0.298 with $95 \% \mathrm{Cl}(0.146-0.609))$ and insufficient staff (aOR=0.388 with $95 \% \mathrm{Cl}(0.190-0.794))$ as barriers were less likely to implement pharmacy-run immunization.

\section{Conclusion:}

Addressing the identified barriers driving pharmacist's lower willingness to vaccinate would be required to fully harness the potential of pharmacists as vaccinators.

\section{Introduction}


Vaccination is one of the most effective methods of combating infectious diseases and has the potential to significantly reduce the spread of many diseases in addition to a substantial reduction in social and economic costs [1]. However, despite the recommendations in favor of vaccinations, the burden of vaccine-preventable diseases remains high in many countries [2]. This could be instigated by low vaccination coverage rates which may also lead to the re-emergence of many diseases even if the disease in question has already been eradicated in a given area $[1,3]$. Thus, ensuring the provision of vaccinations for the most susceptible population groups in the entire community should be one of the top priorities of institutions dealing with public health.

Traditionally, vaccinations have been provided by doctors. However, since the 1990s, some countries such as Canada, Portugal, UK, and the US started utilizing community pharmacists to deliver immunization services and more recently in Australia [4-9]. In the light of lack of accessibility to immunizers and time identified in many studies as barriers to achievement of target immunization coverage, community pharmacists present a valuable resource in supporting the success of immunization programs. They present an opportunity to provide easy access to vaccination services with large flexibility in the time given that they work longer than conventional working hours with no need for appointments [10]. Besides, the literature showed that community pharmacies have wide coverage including more deprived medically underprivileged locations [11] and patients who have not been previously vaccinated by traditional providers as well [12].

However, several barriers to instigating a pharmacist-run immunization program exist. Whilst legislation and healthcare systems across countries differ, there are still common lessons that can be learned about barriers faced during the process of establishing community pharmacy-provided vaccination services [10, $13,14]$.

Community pharmacies in Lebanon are facilities where vaccinations cannot be administered. Pursuant to the law, vaccinations can only be administered by doctors who only can qualify patients for vaccinations. So far, no studies in Lebanon have targeted this topic. Hence, exploring the possibility of expanding the scope of practice of community pharmacists is of critical importance particularly during the COVID-19 pandemic as pharmacists could have a significant impact on the vaccination coverage rate of patients. The success of the pharmacist-run immunization program relies on identifying relevant perceived barriers and overcoming them in a proper and timely manner.

Our survey aims to identify the barriers perceived by Lebanese community pharmacists to implementing a program utilizing pharmacists as immunizers, and the association of these barriers with their willingness to provide such service.

\section{Methods:}

\section{Study design and population:}


A cross-sectional study using an internet-based survey was conducted over the period of time extending between the $1^{\text {st }}$ of November and the end of December 2020. All community pharmacists working currently in a pharmacy setting and who agreed to participate in the study were eligible for participation. Exclusion criteria included: clinical pharmacists, retired community pharmacists, those who were out of the country at the time of the survey, as well as those not practicing actually. Pharmacists who were unreachable due to a change of their contact information during the time of the survey and those who refused to participate in the study were also excluded.

\section{Tool Development}

A 30-items questionnaire was developed and designed specifically by the authors to identify the main barriers perceived by community pharmacists. It was drafted, piloted, and modified with input from community-based pharmacists prior to distribution. A panel of experts from different geographical areas was asked to comment on the clarity, wording, interpretability, and relevance of the items in the survey. An Arabic version of the questionnaire was made based on standard translation guidelines [15]. The questionnaire was pre-tested among 30 community pharmacists for survey flow, functionality, readability, and clarity. Based upon feedback from the pre-test of the questionnaire among 30 community pharmacists, minor modifications were made. The average time for filling the survey was 5 minutes. The questionnaire was self-administered and consisted of open-ended questions. It was divided into 3 domains:

1. Baseline information of participants: including age, gender, profile, educational level, clinical experience, and working hours. Participants were also asked about some pharmacy information (urbanicity, province, opening hours...)

1. Pharmacists' willingness to administer adult vaccines under any circumstances. The term "under any circumstances" refers to pharmacists' willingness to vaccinate in all conditions (whatever during or outside a pandemic period, with or without additional training, with or without a collaborative framework with physicians).

3. Pharmacists' perceived barriers: it comprises 20 items designated to assess elements considered as barriers for the pharmacist-Run immunization service. All the items were answered on a Disagree/Agree basis. An "agree" response was assigned a value of ' 1 ' and a "disagree" a value of ' 0 '. Hence, the cumulative score for all 20 concerns questions would range from 0 to 20 points.

Participants 'overall barriers" score was categorized using modified Bloom's cut-off point, as "high" if the score was between 60 and 100\% (12-20 points), and "low" if the score was less than $60 \%(<12$ points).

\section{Sample size calculation}


In Lebanon, the practice of the profession of pharmacy requires registration with the Order of Pharmacists (OPL). At the time of the study, OPL records indicated a total number of 4185 licensed community pharmacies. Considering a confidence level of $95 \%$, a margin of error of $5 \%$, and an estimated population of 4185 community pharmacies active in Lebanon, the required sample size of this survey would be at least 352; it was calculated using the online RAOSOFT sample size calculator.

\section{Data collection}

As the Lebanese government recommended the public to minimize face-to-face interaction, and this period includes the lockdown during feasts, community pharmacists were electronically invited to participate. An anonymous online questionnaire (Arabic and English versions) using a Google form was sent using WhatsApp or email to all community pharmacists. Then, pharmacists were contacted via phone call and notified about the survey and its purpose. This link of the study included a brief introduction to the background, the objective of the survey, and instructions for filling the questionnaire.

\section{Ethical considerations}

Written informed consent was obtained for each participant. They were reassured that their participation is voluntary and that they were free to withdraw at any time. In addition, all information were gathered anonymously and handled confidentially. The study design assured adequate protection of study participants, and neither included clinical data about patients nor configured itself as a clinical trial.

\section{Statistical analysis}

The collected data was exported to a Microsoft Excel 2016 for cleaning and coding. The cleaned data was exported and analyzed using the statistical software SPSS (Statistical Package for Social Sciences), version 24.0. Descriptive statistics were reported using frequency with percentages for categorical variables. To check scale reliability, Cronbach's alpha was conducted on barriers score. Responses to questions relating to barriers were tabulated in terms of the percentages of "disagree" and "agree" on answers. Bivariate analysis was performed to examine factors associated with the dependent variable (willingness to vaccinate under any circumstances) and the independent variables. The relation between nominal variables was tested using the chi-squared test. The variables in bivariate analysis with $p$-value < 0.2 were entered into multivariable logistic regression. Adjusted odds ratio and their $95 \%$ confidence intervals were reported. Two logistic regressions were performed. The first one was conducted to identify factors associated with pharmacists' high perception of barriers and another one to determine the predictors of the pharmacist's willingness to vaccinate. In each regression, the final logistic regression model was reached after confirming the adequacy of the data using the Hosmer and Lemeshow test. The level of statistical significance was set at a p-value $<0.05$.

\section{Results:}


A total of 412 community pharmacists participated in this survey. Out of the total, 278 (67.48\%) showed their willingness to expand their practice scope to include immunization. They were willing to administer vaccines under any circumstances (Figure 1).

Table 1 summarized the association between baseline information and the pharmacist's willingness to vaccinate. Of the total, $86 \%$ of males were more willing to administer vaccines comparing to females $(52.2 \%), 69.2 \%$ of community pharmacists aged less than 40 years compared to their counterparts aged more than 40 years $(59.5 \%)$. The highest education level seems not associated with the pharmacist's willingness to vaccinate ( $p$-value $>0.05)$. In regards to pharmacist profile, owners $(68.3 \%)$ and pharmacies managers (90.9\%) were more willing to provide vaccines than staff pharmacists (58.9\%). Similarly, pharmacists with large experience (74.4\%) and with previous immunization experience $(72.8 \%)$ had the highest willingness to vaccinate. More than $60 \%$ of community pharmacies located either in rural or urban areas were willing to vaccinate. No association was reported between urbanicity and pharmacist's willingness. However, pharmacists in Mount-Lebanon province showed the highest willingness to extend their practice scope. Notably, this will increase with the number of hours per week pharmacy is open. 
Table 1: Bivariate analysis between baseline information and pharmacist's willingness to vaccinate Willingness to vaccinate under any circumstances

Gender

Total No Yes P-value

Gender

$\mathrm{N} \quad \mathrm{n}(\%) \quad \mathrm{n}(\%)$

Male

Female

$\begin{array}{llll}186 & 26(14 \%) & 160(86 \%) & <0.001 \\ 226 & 108(47.8 \%) & 118(52.2 \%) & \end{array}$

Age (years)

Less than 40 years

$338 \quad 104(30.8 \%) \quad 234(69.2 \%)$

40 years and more

74

$30(40.5 \%) \quad 44(59.5 \%)$

Highest educational level

0.004

BS pharmacy
More than BS (Pharm D, Master, PhD)
Profile

$\begin{array}{lll}229 & 67(29.3 \%) & 162(70.7 \%) \\ 183 & 67(36.6 \%) & 134(63.4 \%)\end{array}$

Profile

$183 \quad 67(36.6 \%) \quad 134(63.4 \%)$

Staff pharmacist

Manager

Owner

Years of experience

\begin{tabular}{lll}
\hline 141 & $58(41.1 \%)$ & $83(58.9 \%)$ \\
\hline 44 & $4(9.1 \%)$ & $40(90.9 \%)$ \\
\hline 227 & $72(31.7 \%)$ & $155(68.3 \%)$
\end{tabular}

0-10 years

More than 10 years

Previous experience in immunization

No

Yes

Pharmacist's working hours per week

0-24 hours

More than 24 hours

Geographical location of the pharmacy

Rural

2




\begin{tabular}{|lllll|}
\hline Urban & 280 & $87(31.1 \%)$ & $193(68.9 \%)$ & \\
\hline Province & & & & 0.036 \\
\hline Akkar \& North & 48 & $19(39.6 \%)$ & $29(60.4 \%)$ & \\
\hline Mount-Lebanon & 155 & $38(24.5 \%)$ & $117(75.5 \%)$ & \\
\hline Beirut & 73 & $26(35.6 \%)$ & $47(64.4 \%)$ & \\
\hline Bekaa \&Baalbeck-Hermel & 42 & $20(47.6 \%)$ & $22(52.4 \%)$ & \\
\hline South\&Nabatyeh & 94 & $31(33 \%)$ & $63(67 \%)$ & \\
\hline Number of hours per week pharmacy is open & & & & \\
\hline Less than 50 hours & 18 & $15(83.3 \%)$ & $3(16.7 \%)$ & \\
\hline $50-120$ hours & 363 & $112(30.9 \%)$ & $251(69.1 \%)$ & \\
\hline 7 days 24/24 & 31 & $7(22.6 \%)$ & $24(77.4 \%)$ & \\
\hline Total & 412 & & & \\
\hline
\end{tabular}

\section{Concerns and barriers perceived by community pharmacists to implementing a Pharmacist-Run Immunization Service}

The main listed barriers by community pharmacists were the conflicts with other professionals who are eligible to vaccinate $(75 \%)$, the liability and malpractices concerns and the reimbursements concerns (49\%). In addition, $44.4 \%$ of participants worried from the time needed for professional development and training required and $32.8 \%$ were concerned about the cost related to such training. Around $40 \%$ of surveyed pharmacists considered that pharmacists were less trusted by patients to provide immunization. Lack of knowledge of adverse events after immunization and vaccines precautions and contraindications were also cited as barriers by participants $41.3 \%$ and $37.1 \%$ respectively. However, only $4.9 \%$ of found that pharmacists were busy and lack of time to administer the vaccine (figure 2).

Of note, among all surveyed community pharmacists, the majority of them disclosed a low perception of barriers and only $8.5 \%$ of them exhibited a high perception of barriers score (Figure 3 ).

\section{Factors associated with level of barriers perceived by community pharmacists}


Table 2 represents the multivariable logistic regression of the factors associated with high perception of barriers. Our results showed barriers perception score was not associated to neither age groups nor profile, educational level, years of experience or pharmacists working hours ( $p$-value $>0.05$ ). However, females were 2.46 times high barriers level more than males (aOR=2.460 with $95 \% \mathrm{Cl}$ (1.876 to 6.909)). In contradiction, community pharmacists who reported previous experience in immunization were less likely to have a high barrier score ( $\mathrm{aOR}=0.250$ with $95 \% \mathrm{Cl}(0.170$ to 0.586$))$ in comparison to those who didn't have the same experience. Similarly, community pharmacists in urban area $(\mathrm{aOR}=0.451$ with $95 \% \mathrm{Cl}$ (0.199 to 0.926$)$ ) and with extensive working hours ( $\mathrm{aOR}=0.244$ with $95 \% \mathrm{Cl}(0.05$ to 1.2$)$ ) were less likely to have a high perception of barriers.

\section{Factors associated with the willingness of pharmacists to vaccinate under any circumstances}

Table 3 represents the multivariable logistic regression of the factors associated with pharmacists' willingness to administrate vaccines under any circumstances. Our results showed that the odds of willingness were not associated to neither educational level, nor urbanicity or geographical location. However, willingness was negatively associated with gender, where the intention to immunize among females was lower than males (aOR=0.211 with $95 \% \mathrm{Cl}(0.116$ to 0.383$)$ ).

Comparing to their counterparts, community pharmacists aged less than 40 years old were 2.518 times more likely to expand their practice scope to immunization (aOR=2.518 with $95 \% \mathrm{Cl}(1.236$ to 5.130$)$ ). Similarly, owner of pharmacies were 1.504 times more likely to immunize than staff pharmacist (aOR=1.504 with $95 \% \mathrm{Cl}$ (1.098 to 2.103)). In addition, the participants with large work experience ((aOR=1.78 with $95 \% \mathrm{Cl}$ (1.087 to 2.993)) and those reporting previous experience in immunization were 1.702 times more willing to provide immunization (aOR=1.702 with $95 \% \mathrm{Cl}(1.092$ to 3.141$)$ ). In respect of pharmacists working hours, those who worked more than 24 hours per week showed 2.622 times highest intention to provide vaccines (aOR=2.622 with $95 \% \mathrm{Cl}(1.447$ to 4.752$)$.

Finally, barriers were negatively associated with pharmacist's willingness to vaccinate. Respondents who perceived reimbursement (aOR $=0.449$ with $95 \% \mathrm{Cl}(0.264-0.765)$ ), lack of pharmacy space (aOR $=0.410$ with $95 \% \mathrm{Cl}(0.158-1.060))$, sharp safety (aOR=0.298 with $95 \% \mathrm{Cl}(0.146-0.609))$ and insufficient resources and staff $(\mathrm{aOR}=0.388$ with $95 \% \mathrm{Cl}(0.190-0.794))$ were less likely to implement vaccination within their pharmacy's settings than their colleagues. 
Table 2: Factors associated with level of barriers perceived by community pharmacists

\section{Perceived Barriers level}

\begin{tabular}{|c|c|c|c|c|}
\hline Low & High & $\begin{array}{l}\mathrm{P} \text { - } \\
\text { value }\end{array}$ & aOr & $\begin{array}{l}95 \% \text { C.I.for } \\
\text { aOr }\end{array}$ \\
\hline (\%) & n (\%) & & & Lower \\
\hline
\end{tabular}

\section{Gender}

Male

Female

Age groups

Less than $40 \mathrm{y}$

40 years and more

Highest education Level

BS pharmacy

More than BS (Pharm D,

Master, PhD)

Profile

Staff pharmacist

Manager

Owner

Years of experience

0-10 years

More than 10 years

Previous experience in immunization

No

Yes

$\begin{array}{ll}76(79.2 \%) & 20(20.8 \%) \\ 301(95.3 \%) & 15(4.7 \%)\end{array}$

Pharmacist's working hours per week

0.674
$<0.001$

179(96.2\%) 7(3.8\%) 1

198(87.6\%) 28(12.4\%)

$2.460 \quad 1.876$

6.909

0.212

0.207

$206(90 \%) \quad 23(10 \%)$

$171(93.4 \%) \quad 12(6.6 \%)$

0.174

124(87.9\%) 17(12.1\%)

$41(93.2 \%) \quad 3(6.8 \%)$

$212(93.4 \%) \quad 15(6.6 \%)$

$206(88.8 \%) \quad 26(11.2 \%)$

$171(95 \%) \quad 9(5 \%)$

0.996

$<0.001$

1

0.250

$0.107 \quad 0.586$

\begin{tabular}{llll}
\hline 0-24 hours & $81(87.1 \%)$ & $12(12.9 \%)$ & \\
\hline More than 24 hours & $296(92.8 \%)$ & $23(7.2 \%)$ & \\
\hline Urbanicity & & & $\mathbf{0 . 0 2 8}$
\end{tabular}




\begin{tabular}{|c|c|c|c|c|c|}
\hline Rural & $115(87.1 \%)$ & $17(12.9 \%)$ & 1 & & \\
\hline Urban & $262(93.6 \%)$ & $18(6.4 \%)$ & 0.451 & 0.199 & 0.926 \\
\hline \multicolumn{2}{|c|}{ Pharmacy opening hours per week } & \multicolumn{2}{|c|}{0.001} & & \\
\hline Less than 50 hours & $9(50 \%)$ & $9(50 \%)$ & 1 & & \\
\hline $50-120$ hours & $341(93.9 \%)$ & $22(6.1 \%)$ & 0.128 & 0.044 & 0.373 \\
\hline 7 days $24 / 24$ & $27(87.1 \%)$ & $4(12.9 \%)$ & 0.244 & 0.050 & 0.588 \\
\hline
\end{tabular}


Table 3: Multivariable logistic regression of the factors associated with the pharmacist's willingness to administer vaccine under any circumstances

Willingness to administer vaccine under any circumstances

$\begin{array}{lllll}\text { No } & \text { Yes } & \text { P-value } & \text { aOr } & \text { 95\% C.l.for aOr } \\ n(\%) & n(\%) & & & \text { Lower }\end{array}$

Gender

Male

$26(14 \%)$

$160(86 \%)$

1

Female

108(47.8\%)

$118(52.2 \%)$

0.211

0.116

0.383

Age groups

$<0.001$

40 years and

$30(40.5 \%)$

$44(59.5 \%)$

1

more

Less than $40 \mathrm{y}$

104(30.8\%)

$234(69.2 \%)$

$2.518 \quad 1.236$

5.130

Profile

0.023

Staff pharmacist

$58(41.1 \%)$

$83(58.9 \%)$

1

Manager

$4(9.1 \%)$

40(90.9\%)

$1.399 \quad 1.022$

3.243

Owner

$72(31.7 \%)$

155(68.3\%)

$1.504 \quad 1.098$

2.103

Years of

experience

\section{0-10 years}

More than 10 years
Previous experience in immunization

No

Yes

Pharmacist's working hours per week

0-24 hours

More than 24

hours

\section{Reimbursement concerns}

No

$46(21.9 \%)$

Yes

$46(49.5 \%)$

$88(27.6 \%)$
$47(50.5 \%)$

231(72.4\%)

$48(50 \%)$

$230(72.8 \%)$

1.702

1.092

3.141

0.019

$<0.001$

1.00

$\begin{array}{lll}1.78 & 1.087 & 2.993\end{array}$


Lack of pharmacy space to store vaccine

$<0.001$

\begin{tabular}{lllllll}
\hline No & $100(27.5 \%)$ & $263(72.5 \%)$ & & 1 & \\
\hline Yes & $34(69.4 \%)$ & $15(30.6 \%)$ & & 0.410 & 0.158 & 1.060 \\
\hline \multicolumn{2}{l}{ Insufficient staff or resources to implement } & $<0.001$ & & & \\
\hline No & $71(21.8 \%)$ & $255(78.2 \%)$ & & 1 & & \\
\hline Yes & $63(73.3 \%)$ & $23(26.7 \%)$ & & 0.388 & 0.190 & 0.794 \\
\hline Sharp safety concerns & & $<0.001$ & & & \\
\hline No & $93(26.4 \%)$ & $259(73.6 \%)$ & & 1 & & \\
\hline Yes & $41(68.3 \%)$ & $19(31.7 \%)$ & & 0.298 & 0.146 & 0.609 \\
\hline
\end{tabular}

${ }^{*} p$-value $<0.05$ is considered significant

\section{Discussion:}

Improvement of vaccination coverage levels among the Lebanese community is required to combating infectious diseases and to counter the negative health consequences associated with a vaccinepreventable disease. Hence, expanding the scope of practice of pharmacists to include immunization could be streamlined to potentially allow optimal opportunities for the community to receive the required vaccination. However, such a process is encountered by many challenges. To the best of our knowledge, this is the first nationwide study representing community pharmacists in Lebanon, aiming to identify barriers to implementing pharmacist-run immunization and, their associated factors and those associated with the pharmacist's willingness to provide vaccines. According to our calculations, the reached sample size was adequate and higher than required, the gender ratio was estimated to be close to the actual ratio, and participants were from different age categories. This information will be used to develop community-level interventions designed to overcome adjustable barriers in order to improve pharmacist-delivered vaccination services if launched in the future.

The main findings of our study were that more than two-thirds of community pharmacists were willing to expand their practice scope to include immunization under any circumstances. This percentage is considered promising. In a study conducted in Canada regarding attitudes and beliefs of the Canadian health care providers, $82 \%$ of pharmacists would support the expansion of the pharmacists' scope of practice to include the provision of vaccines to adults [16].

Despite the demonstrated success and positive impact of vaccines administration by pharmacists, several barriers were revealed to hinder the process, hence affecting the willingness of pharmacists to 
provide vaccination services in their pharmacies. Among those barriers, the surveyed pharmacists pointed out many factors. The foremost issue in launching the pharmacy-based immunization service was the conflicts with other professionals who are eligible to vaccinate. This common challenge was reported in many countries such as Canada and Australia, where a fierce averseness from the physicians toward permitting pharmacists to administer vaccinations. The reasons behind this opposition and lack of support were reasoned that pharmacists were not adequately or effectively trained to do vaccination or to manage adverse effects following vaccine administration [17-19].

Additionally, community pharmacists have also reimbursements concerns. The remuneration arrangements must at least cover the overhead costs a pharmacy incurs for offering pharmacistdelivered vaccination services. If it's not possible, it makes it difficult for some pharmacies to offer such service to the community especially that patients considered that vaccines offered at pharmacies should be offered at a lower cost. Furthermore, surveyed pharmacists were concerned about the time needed and the cost required for professional development and training. Hence, the importance of establishing an immunization certification program well-scheduled in terms of time and cost in order to be accessible by the interested community pharmacists. Similar barriers were reported in a study conducted among community pharmacists in Michigan [20].

It is noteworthy that more than a third of surveyed pharmacists considered that pharmacists were less trusted by patients to provide immunization. This result is in line with a study conducted in Canada that reported that despite the identification of pharmacists as a reliable source of health information, a physician or nurse recommendation to receive or to administer a vaccine is more influential than a pharmacist recommendation [16].

Other common challenges include issues related to lack of knowledge of managing adverse events following immunization and vaccines precautions and contraindications. The lack of relevant training courses was also pointed out by researchers in Saudi Arabia as a barrier to providing vaccinations in pharmacies [21]. Additional factors were also cited as barriers by participants like limited pharmacy staffing, difficulties in having adequate floor space to provide a vaccination service in the community pharmacy premises, lack of time to administer vaccines, and liability concerns. In the UK, similar concerns were revealed about pharmacist's expertise in delivering vaccination, confidentiality and privacy, and the lack of space in pharmacy.

One interesting finding in our study was that the top majority of the surveyed pharmacists showed a low perception of barriers score. Since the highest perception of barriers could lead to reluctance in implementing vaccination, such a result could explain the willingness of more than two-third of community pharmacists to include immunization in their practice.

In respect of factors affecting the pharmacists' perceived barriers levels, our results showed barriers that females were 2.4 times high barriers level more than males This could explain the lowest willingness to administer vaccine among females than males. In contradiction, having previous experience in immunization was a protector factor against the highest perception of barriers. Similarly, community 
pharmacists in the urban areas and with extensive working hours were less likely to have a high perception of barriers.

Our results showed that the odds of willingness were not with the educational level, urbanicity, or geographical location. However, willingness was negatively associated with gender; where the intention to immunize among females was lower than males. Given that higher barrier perception is associated with a decrease in the willingness to provide vaccine; such result was predictable in the light of the association unveiled in this study between being females and higher perception of barriers.

Comparing to their counterparts, community pharmacists aged less than 40 years old were 2.5 times more likely to expand their practice scope to immunization. This could be due to the fact that younger pharmacist is more motivated to take risks and additional steps.

Similarly, owners of pharmacies were 1.5 times more likely to immunize than staff pharmacists. This result could be explained by the fact that the pharmacy owner is the one who had the real decision in incorporating such service in his practice setting, contrary to the staff pharmacists who cannot decide on this matter and have to follow the pharmacy owner's decision.

Notably, the participants with large work experience and those reporting previous experience in immunization were more willing to provide immunization. Such a result was expected given that pharmacist with large experience could face and overcome during his practice many challenging circumstances, so he expressed a lowest perception of barriers.

In respect of pharmacists' working hours, those who worked more than 24 hours per week showed the highest intention to provide vaccines.

Among barriers cited by pharmacists, reimbursement concern, lack of space, sharp safety and lack of pharmacy space and human resources impacted negatively the pharmacist's willingness to vaccinate. Consistently with other studies, community pharmacists who perceived reimbursement as a barrier were less likely to implement vaccination services in their practice [22, 23]. However, the absence of a reasonable financial benefit on the part of pharmacists (other than the cost of the product and administration cost across all provider types), will lead them to lose their interest in expanding their practice scope as it will only present an additional work. Additionally, there is a need to establish a uniformity of remuneration among community pharmacists before implementing immunization-run pharmacies service

Similarly, lack of pharmacy space, sharp safety, and insufficient resources staff were also negatively associated with the willingness of community pharmacists to implement vaccination within their pharmacy's settings than their colleagues.

\section{Limitations}


Several limitations should be acknowledged in the present study. First, our study relies on community pharmacists' self-reported information, which makes it prone to the disadvantages of desirability biases. Furthermore, this online questionnaire might have favored a selection bias since it might only allow the participation of community pharmacists who have access to online resources to participate. Results may not be generalized to community pharmacists in other countries.

\section{Implications:}

Prior to expanding the practice scope of community pharmacists to include the administration of vaccines, there is a need to address community pharmacists' concerns through enhancing their knowledge and skills by implementing well-structured training under the supervision of competent dons. Simultaneously, it is important to target other healthcare providers to find a framework where they could cooperate with pharmacists in order to reduce any conflict of interest. For future research, it is important to explore community pharmacists' knowledge and attitudes related to immunization provision. Exploring barriers perceived by patients towards receiving vaccines in community pharmacies can greatly enrich our information about the current situation of pharmacy practitioners, and what plans and measures are required to overcome these barriers in Lebanon.

\section{Conclusion:}

Several barriers against the provision of vaccines were identified by surveyed community pharmacists. To fully harness the potential and the willingness of pharmacists as vaccinators, the existing barriers and challenges must be addressed, and focus needs to motivate community pharmacists to expand their role through robust evidence of the worth of a pharmacist's vaccination services.

\section{Declarations}

\section{Funding}

No funding was received.

\section{Availability of data and materials}

Data are available from the corresponding authors upon reasonable request.

\section{Consent for publication}

Not applicable.

\section{Ethical approval and consent for publication}

A written informed consent was obtained from participants. They were informed that all information would be gathered anonymously and handled confidentially and that their participation is voluntary. The 
questionnaire was collected only in subjects who expressed consent for study participation. As individual participants cannot be identified based on the presented material, this study caused no plausible harm or stigma to participants. The study design assured adequate protection of study participants, and neither includes clinical data about patients nor configure itself as a clinical trial.

\section{Competing interests}

The authors declare that they have no competing interests.

\section{Authors' contributions}

Conception and design: D.Y, H.H and L.A.A

Analysis and interpretation of the data: D.Y and L.A.A

Drafting of the article: D.Y, H.H and L.A.A

Critical revision of the article for important intellectual content: D.Y, L.A.A and H.H

Final approval of the article: D.Y, L.A.A and H.H

\section{Authors information}

Dalal Youssef, MSc, MPH, RSM, PhD candidate, Ministry of public Health, Preventive medicine department

Linda Abou Abbas, MPH, PhD, Ministry of Public Health, Epidemiological surveillance unit Hamad Hassan, PharmD, PhD, Ministry of Public Health

\section{Acknowledgments}

The authors acknowledge Dr. Mohamad Noureddine and Dr Suzanne Farhat for their assistance in data collection. The authors also thank all community pharmacists who participated in this study.

\section{References}

1. Andre, F.E., et al., Vaccination greatly reduces disease, disability, death and inequity worldwide. Bull World Health Organ, 2008. 86(2): p. 140-6.

2. Turner, H.C., G.E. Thwaites, and H.E. Clapham, Vaccine-preventable diseases in lower-middleincome countries. Lancet Infect Dis, 2018. 18(9): p. 937-939. 
3. Klepac, P., et al., Six challenges in the eradication of infectious diseases. Epidemics, 2015. 10: p. 97-101.

4. Hook, S. and J. Windle, Community pharmacy influenza immunisation increases vaccine uptake and gains public approval. Aust N Z J Public Health, 2013. 37(5): p. 489-90.

5. Anderson, C. and R. Sharma, Primary health care policy and vision for community pharmacy and pharmacists in England. Pharm Pract (Granada), 2020. 18(1): p. 1870.

6. Raiche, T., et al., Community pharmacists' evolving role in Canadian primary health care: a vision of harmonization in a patchwork system. Pharm Pract (Granada), 2020. 18(4): p. 2171.

7. Ribeiro, N., et al., Primary health care policy and vision for community pharmacy and pharmacists in Portugal. Pharmacy Practice (Granada), 2020. 18.

8. Dineen-Griffin, S., S. Benrimoj, and V. Garcia-Cardenas, Primary health care policy and vision for community pharmacy and pharmacists in Australia. Pharmacy Practice, 2020. 18.

9. Salgado, T.M., et al., Primary healthcare policy and vision for community pharmacy and pharmacists in the United States. Pharmacy Practice (Granada), 2020. 18.

10. Capurso, K.A. and M.F. Powers, Barriers to Implementing a Pharmacist-Run Immunization Service, as Perceived by Pharmacists, in a Community Pharmacy Chain. Journal of Pharmacy Technology, 2006. 22(2): p. 91-94.

11. Thomson, K., et al., The effects of community pharmacy-delivered public health interventions on population health and health inequalities: A review of reviews. Prev Med, 2019. 124: p. 98-109.

12. Murphy, P.A., et al., Pharmacy provision of influenza vaccinations in medically underserved communities. J Am Pharm Assoc (2003), 2012. 52(1): p. 67-70.

13. Srivastav, A., et al., U.S. clinicians' and pharmacists' reported barriers to implementation of the Standards for Adult Immunization Practice. Vaccine, 2018. 36(45): p. 6772-6781.

14. Bach, A.T. and J.A. Goad, The role of community pharmacy-based vaccination in the USA: current practice and future directions. Integr Pharm Res Pract, 2015. 4: p. 67-77.

15. Beaton, D., et al., Guidelines for the Process of Cross-Cultural Adaption of Self-Report Measures. Spine, 2001. 25: p. 3186-91.

16. MacDougall, D., et al., Routine immunization of adults by pharmacists: Attitudes and beliefs of the Canadian public and health care providers. Hum Vaccin Immunother, 2016. 12(3): p. 623-31.

17. Goodyear-Smith, F., et al., Immunization champions: characteristics of general practitioners associated with better immunization delivery. Hum Vaccin, 2009. 5(6): p. 403-11. 
18. Camilleri, S.A., et al., Predictors of yearly influenza vaccination in hospitalized and community based patients. Multidiscip Respir Med, 2018. 13: p. 23.

19. Gerges, S., et al., Pharmacists as vaccinators: An analysis of their experiences and perceptions of their new role. Hum Vaccin Immunother, 2018. 14(2): p. 471-477.

20. Kelling, S.E., et al., Cross-Sectional Survey of Perceived Barriers Among Community Pharmacists Who Do Not Immunize, in Wayne County, Michigan. Infect Dis Ther, 2016. 5(4): p. 525-533.

21. Balkhi, B., et al., Readiness and willingness to provide immunization services: a survey of community pharmacists in Riyadh, Saudi Arabia. Safety in Health, 2018. 4(1): p. 1.

22. Skelton, J.B., Pharmacist-provided immunization compensation and recognition: white paper summarizing APhA/AMCP stakeholder meeting. J Am Pharm Assoc (2003), 2011. 51(6): p. 704-12.

23. Hartzell, V., Reimbursement for service of administering vaccines, from an independent pharmacy perspective. Pharmacy Times. Available at: https://www.pharmacytimes.

com/publications/supplementals/2017/ImmunizationSupplementJune2017/reimbursement-forserviceof-administering-vaccines-from-an-independent-pharmacy-perspective. (accessed Jul 19, 2020), 2017.

\section{Figures}

\section{Figure 1: Community Pharmacist's willingness to administer vaccine under any circumstances $(\mathrm{N}=\mathbf{4 1 2})$}

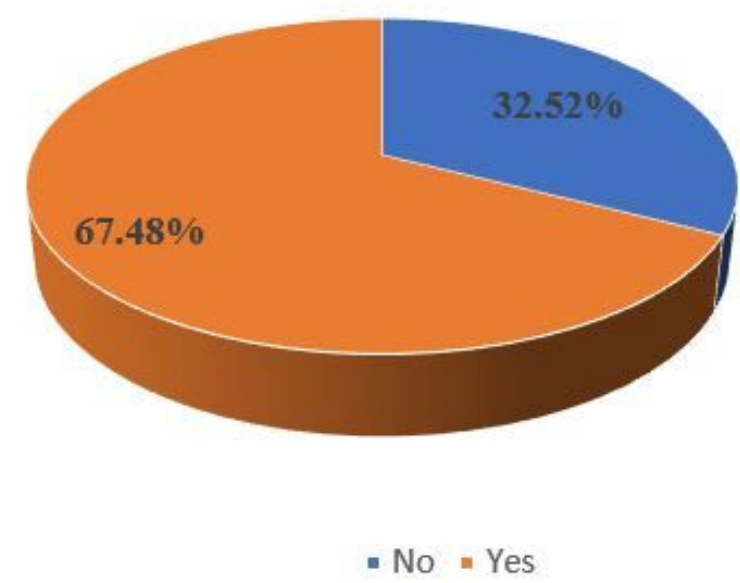

Figure 1

Community Pharmacist's willingness to administer vaccine under any circumstances $(N=412)$ 


\section{Figure 2: Concerns and barriers perceived by community pharmacists about incorporating vaccine administration into practice}

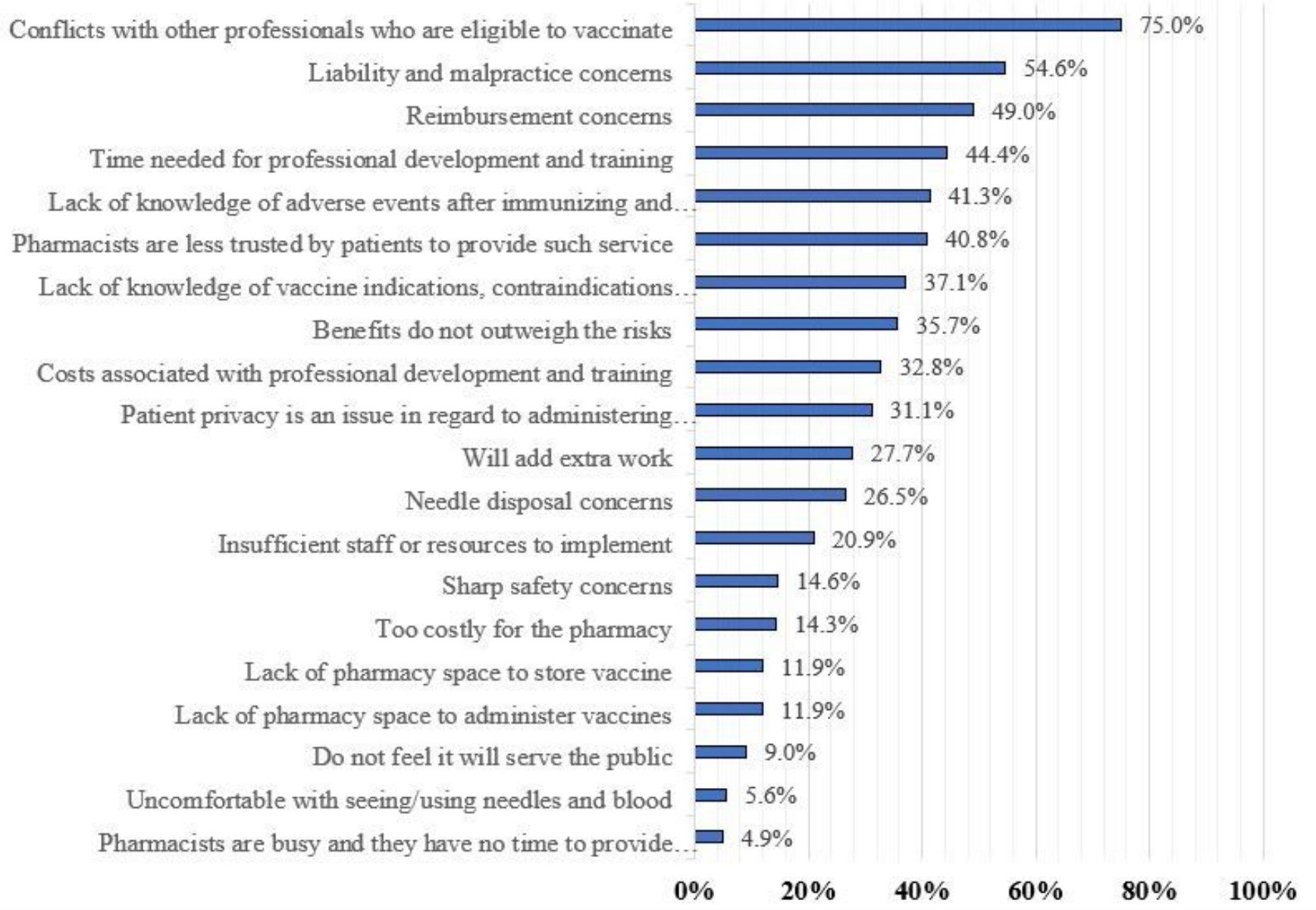

\section{Figure 2}

Concerns and barriers perceived by community pharmacists about incorporating vaccine administration into practice

\section{Figure 3: Perceived overall barriers score}

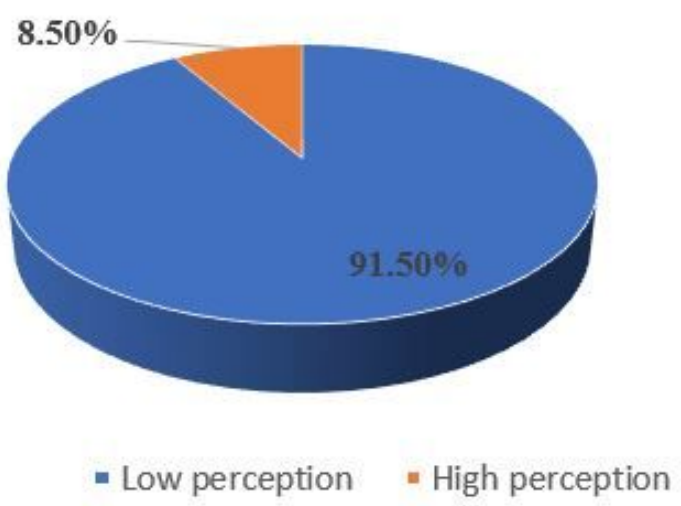


Figure 3

Perceived overall barriers score 